\title{
Recuperación y análisis de los fondos archivísticos de la Real Academia de Bellas Artes de San Fernando: el taller de encuadernación de Joaquín Ibarra
}

Recovery and analysis of archival collections of the Royal Academy of Fine Arts of San Fernando: the binding workshop of Joaquín Ibarra

\section{Antonio Carpallo Bautista (1), Yohana Yessica Flores Hernández (1), Marisa Moro Pajuelo (3) \\ (1) Universidad Complutense de Madrid, Facultad de Ciencias de la Documentación, C/ Santísima Trinidad 37, 28010 Madrid, España, acarpall@ucm.es , amoxtlan@gmail.com (3) Real Academia de Bellas Artes de San Fernando, C/ Alcalá 13, 28014 Madrid, España, biblioteca@archivobiblioteca-rabasf.com}

\begin{abstract}
Resumen
El presente estudio tiene como objetivo principal poner en valor el trabajo realizado por el impresor y encuadernador zaragozano Joaquín Ibarra y Marín en la Real Academia de Bellas Artes de San Fernando, con taller en Madrid desde 1754 hasta su muerte en 1785. Se ha estudiado la documentación de archivo donde se indica que Ibarra trabajó para la Real Academia desde 1771 hasta 1784. Se han consultado los libros de cuentas y legajos del Archivo de la Academia y analizado in situ las encuadernaciones reseñadas en la documentación. Los resultados obtenidos indican los tipos de encuadernaciones encargadas, sus precios, materiales, técnicas de construcción y decoración, destinatarios de las encuadernaciones, relaciones con otros libreros y encuadernadores, ayudando así a entender mejor la historia de la propia institución, al gremio de libreros, impresores y encuadernadores madrileños y, por consiguiente, de la sociedad madrileña de la época. Como conclusión podemos determinar que Joaquín Ibarra compaginó su tarea de impresor con la de encuadernador, aunque a una escala menor, con trabajos modestos (encuadernaciones en pergamino, holandesa, rústica) sin realizar encuadernaciones de lujo, trabajos que derivaba a otros talleres de encuadernación como el de Sancha. Sería recomendable continuar con esta investigación y estudiar los trabajos de Ibarra en otras Reales Academias y así constatar la presencia del mismo tipo de trabajos y precios, entre otros aspectos.
\end{abstract}

Palabras clave: Edad Moderna. Real Academia de Bellas Artes de San Fernando. Ibarra y Marín ,Joaquín. Impresores. Encuadernadores. Encuadernación histórica. España.

\section{Introducción}

Joaquín Ibarra y Marín nació en Zaragoza el 19 de julio de 1725. Aprendió de su tío Manuel Ibarra, impresor y encuadernador de la Universidad de Cervera, donde estudió Humanidades. Con

\begin{abstract}
The main objective of this study is to highlight the work done by the printer and bookbinder Joaquín Ibarra y Marín in the Royal Academy of Fine Arts of San Fernando, with a workshop in Madrid from 1754 until his death in 1785 . The objectives are they have already reached the archive documentation where it is indicated that Ibarra worked for the Royal Academy from 1771 to 1784 . The methodology has been carried out by consulting the accounts books and files of the Academy Archive and the analysis in situ of the bindings outlined in the documentation. The results obtained indicate that we have known the types of bindings commissioned, their prices, materials, construction and decoration techniques, recipients of the in-frames, relationships with other booksellers and in-notebooks, thus helping to better understand the history of the institution itself, the guild of booksellers, printers and bookbinders from Madrid and therefore the Madrid society of the time. As conclusions we can determine that Joaquín Ibarra combined his task of printer with that of bookbinder, albeit on a smaller scale, to perform modest works (bindings in parchment, Dutch, rustic) without making luxury bindings, works that led to other bookbinding workshops like Sancha's. It would be advisable to continue with this research and study the work done by this bookbinder in other Royal Academies and thus verify if he performed the same type of work, prices, among other aspects.
\end{abstract}

Keywords: Modern Age. Royal Academy of Fine Arts of San Fernando. Ibarra y Marín, Joaquín. Printers. Binders. Historical binding. Spain.

19 años, en 1754 marcha a Madrid para establecer su propio taller en la calle de las Urosas y después se trasladaría a la calle Gorguera donde trabajaría hasta su muerte el 13 de noviembre de 1785. 
Ibarra destacó en las Artes del Libro, sobre todo en la impresión, con obras tan importantes como el Quijote y el Salustio. De su taller salieron muchas encuadernaciones corrientes, en pergamino, holandesa, en pasta y en rústica, pero también algunas de lujo, aunque muchas de sus obras impresas fueron encuadernadas por otros artífices siguiendo las patrones establecidos en la Imprenta Real y en la Compañía de Libreros del Reino (Castañeda y Alcover, 1958, p. 130133) como, por ejemplo, las del taller de Antonio de Sancha.

Fue impresor de Cámara de S. M., del Ayuntamiento de Madrid desde 1772 y de la Real Academia Española, nombrado en junio de 1779, y habitual de la Real Academia de Bellas Artes de San Fernando. A su muerte continuaron con la imprenta su viuda y su hija Manuela hasta la muerte de su viuda en 1805. En 1820 el Ayuntamiento de Madrid nombra Impresor y Librero de la villa a Agustín Íñiguez e Ibarra, nieto de Joaquín, pero en 1836 la imprenta desaparece y sus utillajes son vendidos y dispersados entre diferentes talleres de impresión madrileños.

En cuanto a la relación entre Ibarra y la Real Academia de Bellas Artes de San Fernando destaca sobre todo su labor de impresor aunque también encontramos encargos de encuadernaciones modestas en pergamino.

\section{Objetivos y metodología}

Este trabajo forma parte de los resultados de los Proyectos de investigación I+D (MINECO), titulado "La encuadernación española en las Reales Academias: encuadernadores, talleres y tipologías ligatorias (S. XVIII-XX)" (HAR2017-83387P) (2018-2021) y del Proyecto financiado por la Universidad Complutense y el Banco Santander dentro de los proyectos 2016-2017 con el título "Las encuadernaciones de la Real Academia de Bellas Artes de San Fernando: estudio, digitalización, identificación y difusión" (PR26/16-20257), junto a las cláusulas incluidas en el convenio firmado entre la Real Academia de Bellas Artes de San Fernando y el grupo Bibliopegia de la Universidad Complutense de Madrid en 2012, para el estudio de las encuadernaciones de la Biblioteca, Archivo, Museo y Calcografía Nacional.

El estudio comenzó con la confección de un inventario de todas las encuadernaciones artísticas de los diferentes departamentos, haciendo un análisis detallado de los materiales empleados, técnicas de construcción, estructura, técnicas y elementos decorativos y estado de conservación, además de identificar los antiguos poseedores y sobre todo los encuadernadores; también se digitalizaron las encuadernaciones inventariadas.
De forma paralela se revisaron los libros de cuentas y legajos del Archivo, con el fin de obtener información sobre los gastos de la Academia, desde su creación, en encuadernaciones, teniendo en cuenta que las obras que proceden de legados no aparecerán en esta documentación.

En los recibos encontramos informaciones muy interesantes como quién, el Bibliotecario o el Conserje, realiza el encargo al impresor-encuadernador Joaquín Ibarra, fecha de la solicitud, el detalle de los libros que se mandan a encuadernar u otros encargos como carteras, pliegos de papel, entre otros, con la cantidad que se debe abonar por ejemplar o trabajo realizado, y el recibí con la fecha del pago y con las firmas dando el visto bueno de la Junta Particular y/o el Conserje junto a la firma de Ibarra o su viuda a partir de 1785 constatando que ha recibido la cantidad acordada.

Una vez extraída la información sobre los pagos abonados a Ibarra, se intentan localizar, mediante la escasa información que suministran los recibos, las obras en el depósito de la Biblioteca, labor bastante difícil ya que muchas han desaparecido con el tiempo. Localizada la obra, se digitaliza la encuadernación y se realiza un análisis de los aspectos más relevantes como materiales (pieles, papeles decorados...), técnicas de construcción (tipos de costuras...) y elementos decorativos, todo ello muy importante para el establecimiento de un corpus de hierros (florones, paletas y ruedas), y posterior comparación con otras encuadernaciones de la Academia o de otras instituciones donde pudiera haber trabajado el encuadernador.

\section{Estudio de los recibos y anotaciones sobre encargos de encuadernaciones: 1771-1779}

Se advierte una relación comercial entre Joaquín Ibarra y Antonio de Sancha en torno a la impresión y encuadernación de ejemplares para la Academia de Bellas Artes. El primer apunte que encontramos con esa relación es de 1771 (Libro Tesorero. Libro de cuentas, sig. 3-214, recibo $\mathrm{n}^{\circ} 22$ y 23) cuando se encarga a Ibarra la impresión de la Relación de premios, esquelas y catálogo de individuos de la Academia por una cuantía de 7.575 $r^{s} v^{n}$; al mismo tiempo se observa el apunte a favor Antonio de Sancha donde se encarga la encuadernación de la Relación de premios, junto a otros trabajos menores, por un montante de $3.622 \mathrm{r}^{\mathrm{s}} \mathrm{v}^{\mathrm{n}}$.

En la nota del 27 de diciembre de 1771 (Libro Tesorero. Libro de cuentas, sig. 3-214, recibo $n^{\circ} 1, h$. 17) (Figura 1) se pueden apreciar apuntes de trabajos realizados de impresión y encuadernación de la Oración de la Real Academia de S. Fernando al Rey Nuestro Señor con motivo del feliz nacimiento del Infante por Ibarra, como la entrega 
de cinco pliegos de molde a $20 r^{s} v^{n}$ cada uno; una tirada de 780 ejemplares en papel fino; la impresión en papel de "Holanda" de 50 ejemplares a 6 $r^{s} v^{n}$ cada uno; la encuadernación de 684 ejemplares en papel pintado a $1 / 2 r^{s} v^{n}$ cada uno; la encuadernación de 18 ejemplares en tafilete con papel de "Holanda" a $60 r^{5} v^{n}$ cada uno (Figura 2); la encuadernación de 6 ejemplares en pasta con papel de "Holanda" a $14 r^{\mathrm{s}} \mathrm{v}^{\mathrm{n}}$ cada uno; la encuadernación de 94 ejemplares en pasta con papel regular a $10 r^{5} v^{n}$ cada uno; y la impresión de 2.000 esquelas batidas y cortadas, todo ello por un montante de $4.409,22 r^{s} v^{n}$ y que firma como recibido Joaquín Ibarra el 30 de diciembre de 1771. Un ejemplo de estos recubrimientos es una encuadernación holandesa (sig. C-859) en tafilete color marrón con puntas y papel marmoleado tipo plegado español, de colores verde, gris, rojo y negro, con el lomo liso y la tapa suelta, una costura a la americana, con ocho nervios y ocho estaciones de costura, careciendo de tejuelo y de cabezadas. También encontramos la impresión de ejemplares en papel fino (sig. F-8657, F-8658, F-11663) cortados.

Además, localizamos anotaciones del Conserje de la Academia, tanto de impresiones como de encuadernaciones. Un ejemplo lo encontramos en el año 1776 (Legajo Tesorero. Cuentas del conserje, sig. 1-10-3), donde se indica la entrega por el impresor Ibarra, el 9 de noviembre, de veinticinco juegos en papel a $8,2 r^{5} v^{n}$ cada volumen, de la obra Principios de matematica: donde se enseña la especulativa, con su aplicacion a la dinámica, hydrodinámica, óptica, astronomía, geografía, gnómica, arquitectura, perspectiva y al calendario, de Benito Bails. Otra nota del mismo documento indica la entrega de catorce ejemplares en pasta junto a ocho en tafilete.

En el mismo legajo, en un documento del 24 de julio de 1779, aparece una nota donde se indica que Joaquín Ibarra ha enviado a la Academia 36 paquetes de 40 ejemplares a $2 r^{s} v^{n}$ cada uno, más 29 ejemplares sin estampar, haciendo un total de 1.500 ejemplares, todo ello del tomo segundo de Elementos de matematica de Bails que la Academia envía a encuadernar. Del tomo tercero Ibarra envía a la Academia, el 14 de agosto, 100 ejemplares en rústica, tres paquetes con 32 ejemplares cada uno y otro con 30 , lo que suma 94 ejemplares, otros 30 paquetes de 42 ejemplares y uno de 41 , lo que suma 1.301 ejemplares, junto a cinco que faltan por entregar, sumando todo 1.500 ejemplares impresos y encuadernados. Del tomo primero Ibarra envía a la Academia, el 18 de agosto de 1779, 100 ejemplares en rústica, junto a 33 paquetes de 35 ejemplares, otro paquete de 33 ejemplares, otros cuatro paquetes de 42 ejemplares y otro paquete de 39 ejemplares sin estampar, haciendo un total de 1.495 ejemplares del tomo primero.
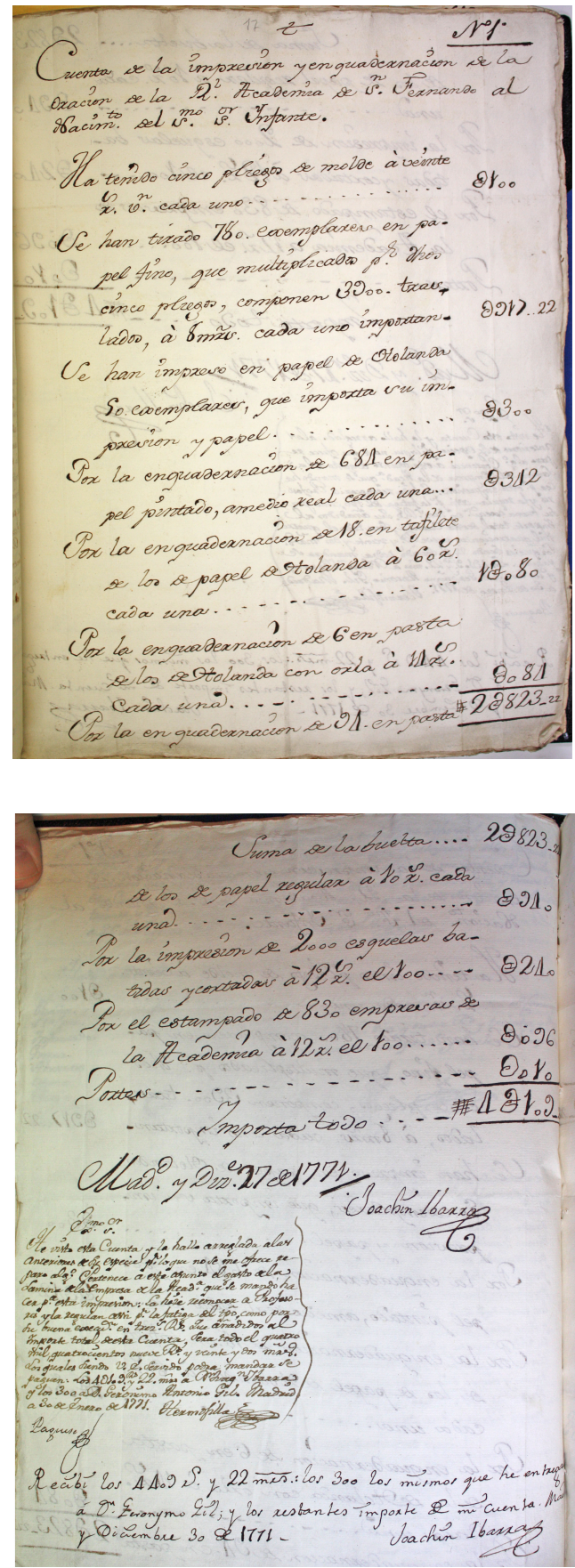

Figura 1. Libro Tesorero. Libro de cuentas. Sig. 3$214, n^{\circ} 1, h .17$

Se puede observar como la Academia confía a Ibarra la impresión de los tres volúmenes de la obra Elementos de matematica de Bails junto a la encuadernación de 100 ejemplares en rústica. De estos 100 ejemplares la nota incluye en qué fecha fueron entregados a la Academia: el día 18 de agosto se entregaron tres juegos, los días 19 y 20 
seis juegos cada uno, el día 21 veinte juegos, el día 26 siete juegos, el día 28 ocho juegos, el 31 de agosto veinte juegos, el 14 de septiembre 25 juegos y por último el 18 de ese mismo mes un juego, haciendo un total de 98 juegos entregados. Se indica también a quien iban dirigidos algunos de los juegos de esta obra, ya encuadernada.

\section{Estudio de los recibos y anotaciones sobre encargos de encuadernaciones: 1780-1784}

El 10 de septiembre de 1779 se entregan tres juegos en rústica de la obra de Bails, uno al Viceprotector de la Academia, el marqués de la Pimentel Florida, Pedro Pimentel, otro al marqués de la Santa Cruz, José Bazán Silva y el tercero al Secretario de la Academia, Antonio Ponz. También se reservan tres juegos para la Academia con fecha del 5 de octubre, y un juego en rústica para la condesa de Pernia, con fecha de 3 de enero de 1780.

En otro documento del mismo legajo se menciona como el 23 de mayo de 1780 Joaquín Ibarra envía a la Academia 486 ejemplares del tomo cuarto Elementos de matematica de Bails, en 12 paquetes de 35 ejemplares y otro paquete de 16 ejemplares, que junto a los 50 tomos que ya había entregado suman los 486 ejemplares.

En el Libro de cuentas 3-222 (h. 15), en el apartado de Cargo de enseres, se indica que el 19 de junio de 1780 Joaquín Ibarra entrega a la Academia 1.492 ejemplares en papel del cuarto tomo del "curso grande" de Bails, es decir la obra Elementos de matematica, teniendo la Academia un total de 5.606 ejemplares.

El 19 de junio Ibarra entrega a la Academia cuatro tomos en rústica, el 20 de mayo había entregado 50 ejemplares en papel; el día 3 de de mayo entrega tres ejemplares en rústica y uno en becerro; el día 23 de mayo entrega 23 paquetes con 35 ejemplares y otro paquete con 16 ejemplares sin estampar, todo ello del cuarto tomo de Elementos de matematica de Bails.

El 2 de marzo de 1781, continua la misma nota del legajo 1-10-3, envía Ibarra 36 paquetes de 40 tomos y otro paquete de 22 ejemplares, todo del tomo quinto de Elementos de matematica de Bails. Ibarra señaló que faltaban por entregar 38 ejemplares, haciéndolo el día 10 de marzo, de los cuales: 30 tomos fueron a la Academia, cuatro se los quedó el propio autor Benito Bails, uno para el conde de Pernia, Pelayo Pernia Girón Castillo, otro a Pedro Silva Bazán Sarmiento y el último al conde de Floriblanca, José Moñino Redondo.

En el mismo legajo, con fecha del 19 de enero de 1782 , encontramos una nota donde se menciona la entrega por parte de Ibarra, de la impresión del tomo sexto de la obra de Bails, entregados en 34 paquetes de 40 ejemplares, junto a tres paquetes de 40 ejemplares ya confiados al encuadernador Manuel Millana y ocho ejemplares sueltos.
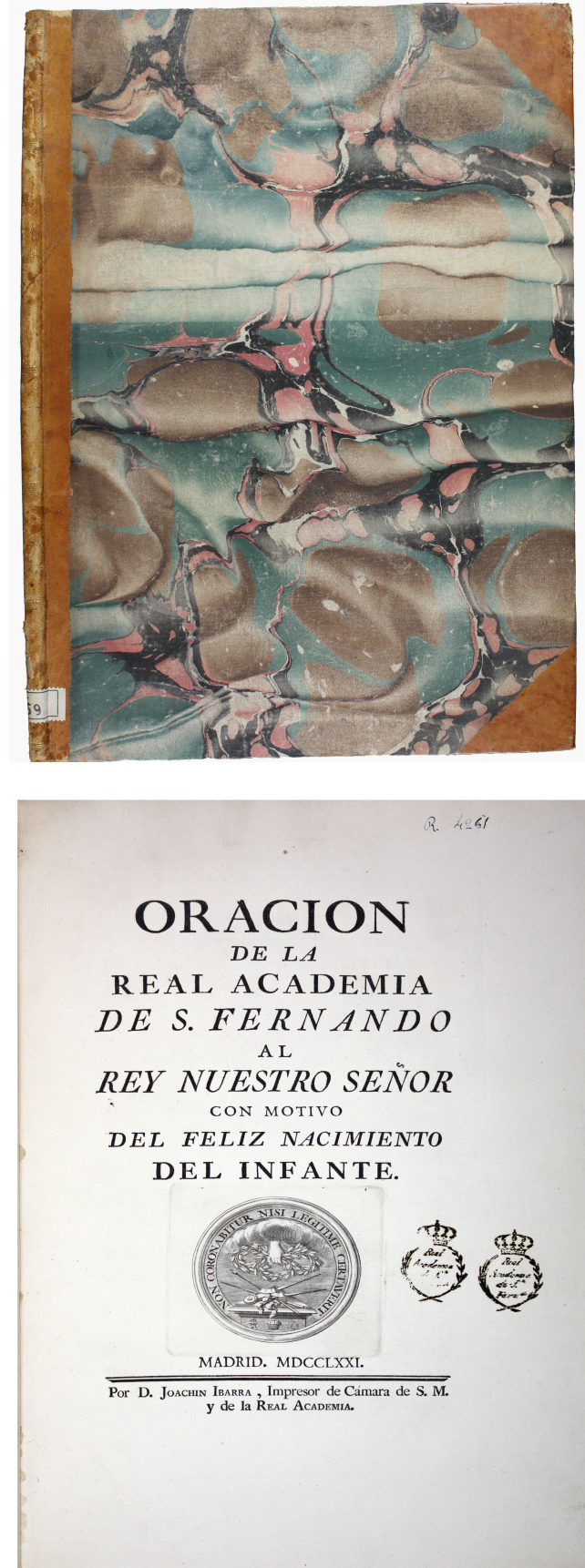

Figura 2. Lomo, tapa anterior y portada (sig. C-859)

En el Libro de cuentas 3-223 (h. 15), en el "Resumen de los cargos antecedentes" se indica que el 19 de enero de 1782 la Academia tenía 5.195 ejemplares del "curso grande" de Bails, de los cuales 1.488 eran del tomo sexto y que la imprenta de Ibarra había entregado a la Academia, 
tomos Principios de matematica: donde se enseña la especulativa, con su aplicacion a la dinámica, hydrodinámica, óptica, astronomía, geografía, gnómica, arquitectura, perspectiva y al calendario, del año 1776, con sucesivas impresiones de cada tomo, incluida la encuadernación en alguna ocasión, y del "curso grande" en once tomos Elementos de matemática, impresos entre 1779 y 1796. También encontramos impresiones de la Relación de Premios, encuadernados en varias ocasiones por Antonio de Sancha.

Observamos la gran cantidad de ejemplares que se imprimían, alrededor de 1.500 por tomo de las obras de Bails, los diferentes tipos de encuadernaciones (holandesas, pergamino, pasta, tafilete, rústica...), todas ellas sencillas y modestas, con diferentes precios, destinadas sobre todo a los alumnos de la Academia.

Otro dato a resaltar son los juegos de obras que se destinaban a la Biblioteca de la Academia y los que se regalaban a personalidades de la nobleza como al Viceprotector de la Academia, el marqués de la Pimentel Florida, Pedro Pimentel, al marqués de la Santa Cruz, José Bazán Silva, la condesa de Pernia y el conde de Pernia, Pelayo Pernia Girón Castillo y el conde de Floriblanca, José Moñino Redondo, junto a ministros y personalidades de la propia Academia.

\section{Referencias}

Acín Fanlo, José Luis; Murillo López, Pablo (dir.) (1993). Joaquín Ibarra y Marín, impresor, 1725-1785. Zaragoza: Ibercaja: Diputación General de Aragón, 1993.

Amado Franco, Lahoz [et al.] (2006). Fundiendo los plomos: homenaje al tipógrafo aragonés Joaquín Ibarra y Marín. Zaragoza: Foro de Diseño: Asociación Aragonesa de Profesionales del Diseño Gráfico: Ibercaja, 2006.

Bermejo Martín, José Bonifacio (dir.) (1998). Enciclopedia de la encuadernación. Madrid: Ollero \& Ramos, 1998.

Blanco-Belmonte, Marcos Rafael (1931). El maestro Ibarra: homenaje que la Casa Gans, al celebrar sus bodas de oro, dedica al gran impresor Joaquín Ibarra. 1931.

Blanco-Belmonte, Marcos Rafael (2014). Un día en la vida del maestro impresor Joaquín Ibarra. Madrid: Turpin, 2014.

Buonocore, Domingo (1976). Diccionario de Bibliotecología Buenos Aires: Marymar, 1976.

Capela Martínez, Miguel (1963). Las artes gráficas de Madrid en el siglo XVIII. // La industria en Madrid: ensayo histórico crítico de la fabricación y la artesanía madrileñas: siglos XVIII al XIX desde 1701 a 1912: la industria en Madrid en el siglo XVIII. Madrid: Artes Gráficas y Ediciones, 1963. 121-155.

Carpallo Bautista, Antonio (2012). Encuadernaciones del siglo XVIII en la Catedral de Toledo. Madrid: Ollero y Ramos, 2012.

Carpallo Bautista, Antonio [et al.] (2015). Encuadernaciones de las Guías de Forasteros de la Real Academia de la Historia. Madrid: Ollero y Ramos, 2015.
Carrión Gútiez, Manuel (1994). La encuadernación española. // Historia ilustrada del libro español: De los incunables al siglo XVIII. Madrid: Pirámide, 1994. 95-446.

Castañeda y Alcover, Vicente (1958). Ensayo de un diccionario biográfico de encuadernadores españoles. Madrid: Maestre, 1958.

Cavestany, Julio (1927). Las industrias artísticas madrileñas en la exposición del antiguo Madrid 1926-1927... Madrid: Gráficas Reunidas, 1927.

Cotarelo y Mori, Emilio (1923). Homenaje a Joaquín Ibarra: Sesión celebrada en la Plaza de la Villa el 21 de julio de 1923. Madrid: Imprenta Municipal, 1923.

Donoso-Cortés y Mesonero-Romanos, Ricardo (2007). Joaquín Ibarra y Marín y su familia: (en cincuenta y tres partidas sacramentales y doce testamentos). Madrid: Instituto de Estudios Madrileños, 2007.

Flores Hernández, Yohana Yessica; Carpallo Bautista, Antonio; Burgos Bordonau, Esther (2018a). El taller de Sancha en la Real Academia de Bellas Artes de San Fernando. // Titivillus. 4 (2018) 39-62.

López Castán, Ángel (1986a). La encuadernación madrileña y la comunidad de mercaderes y encuadernadores de libros de la Corte en el siglo XVIII. // Villa de Madrid. XXIV: 89-90 (1986) 41-63.

López Serrano, Matilde (1937a). La encuadernación en Madrid en la primera mitad del siglo XVIII. // Archivo Español de Arte y Arqueología. 37 (1937) 1-13.

López Serrano, Matilde (1940a). La encuadernación madrileña en la época de Fernando VI. // Archivo Español de Arte. 40 (1940) 27-38.

López Serrano, Matilde. (1945a). La encuadernación española del siglo XVIII. // Gráficas. 17 (1945) 4-5, 28.

López Serrano, Matilde. (1945a). La encuadernación madrileña durante el reinado de Carlos III. // Archivo Español de Arte. 67 (1945) 1-16.

López Serrano, Matilde (1945a). El encuadernador Gabriel Gómez Martín. // Revista de Bibliografía Nacional. VI (1945) 51-72.

López Serrano, Matilde (1946a). Antonio de Sancha, encuadernador madrileño. // Revista de Bibliotecas, Archivos y Museos Municipales. 54 (1946) 269-307.

López Serrano, Matilde (1946a). Una tormenta doméstica en la familia Sancha. // Revista de Bibliografía Nacional. VII (1946) 391-399.

López Serrano, Matilde (1950a). La encuadernación madrileña en la época de Carlos IV. // Archivo Español de Arte. 90 (1950) 115-131.

López Serrano, Matilde (1975). Gabriel de Sancha: editor, impresor y encuadernador madrileño (1746-1820). Madrid: Ayuntamiento, Instituto de Estudios Madrileños.

Moral Sandoval, Enrique (1995). Algunas noticias sobre el impresor aragonés Joaquín Ibarra y Marín. Madrid: Libris.

Ruiz Lasala, Inocencio (1968). Joaquín Ibarra y Marín (17251785). Zaragoza: [s.n.].

Vélez Celemín, Antonio (2017). El marmoleado: del papel de guardas a la obra de arte. Madrid: Antonio Vélez, 2017.

Enviado: 2018-02-07. Segunda versión: 2018-06-05 Aceptado: 2018-06-05

Carpallo, A.; Flores, Y. Y.; Moro, M. Recuperación y análisis de los fondos archivísticos de la Real Academia de Bellas Artes de San Fernando: el taller de encuadernación de Joaquín Ibarra. // Ibersid. 12:2 (2018) 91-96. ISSN 1888-0967. 